\title{
1. Angewandte Geographie - Stellenwert in der Raumplanung
}

\section{Die Bedeutung der Geographie für die Entwicklung der Raumplanung in der Schweiz}

Angewandte Geographie ist in unserem Land eng mit der Geschichte der Raumplanung verbunden. Es lohnt sich deshalb, kurz auf die Entwicklung dieser Staatsaufgabe einzugehen, um die Rolle, welche die Geographie dabei spielte, zu analysieren. Drei Phasen lassen sich da unterscheiden: In einer ersten, längeren Periode stand die «Landesplanung» im Vordergrund, eng verknüpft mit der politischen Auseinandersetzung um eine Neukonzeption des Bodenrechtes. Daran schloß sich eine zweite Phase an, die ganz im Zeichen der Erarbeitung eines Bundesgesetzes über die Raumplanung stand. Nach dessen Ablehnung durch Volk und Stände folgte die dritte, heute noch währende Periode, die mit einer grundlegenden Neuorientierung in der Raumplanung begann und im heute rechtskräftigen Raumplanungsgesetz eine bedeutende Wegmarke überschritt. Gegenwärtig stehen wir mitten im Vollzug dieses Gesetzes.

Das Objekt der Geographie ist «die Landschaft» oder «der Raum». Die Geographie versucht, die Zusammenhänge im Wechselspiel Mensch - Natur aufzudekken und darzustellen - Grundlage auch jeder raumplanerischen Tätigkeit. Es ist darum nicht verwunderlich, daß zu den Pionieren der Schweizer Raumplanung neben Architekten und Kulturingenieuren insbesondere auch Geographen gehörten. Heinrich Gutersohn und Ernst Winkler sind wohl, ohne die Leistung der übrigen Geographen herabzumindern, die bedeutendsten Vertreter ihres Faches in der frühen Raumplanung. Sie waren mitverantwortlich, daß die Raumplanung aus ersten Ansätzen («Binnenkolonisation») der dreißiger Jahre herauswuchs und rund eine Generation später als «Landesplanung» zu einem der ganz großen Diskussionsthemen der schweizerischen Öffentlichkeit wurde. In dieser Zeit, der späten fünfziger und frühen sechziger Jahre, erhitzte zugleich die Debatte um die Neukonzeption des Bodenrechtes die Gemüter. Die Auseinandersetzungen gipfelten in der Volksabstimmung über die Bodenrechtsinitiative der Sozialdemokraten - und fortan gewann die Landesplanung Oberhand. "Mit bedrückender Eindringlichkeit zeigen sich überall unerwünschte Folgen der stürmischen Bevölkerungszunahme und der vielerorts ungeregelten Ausweitung bisheriger Bau- gebiete. Gewässerverschmutzung, Luftverunreinigung, Belästigung durch Lärm, ungelöste Verkehrsprobleme und ähnliches machen den Behörden zu schaffen; dazu kommt aber auch eine durch Verzettelung der Bauten unrationelle Nutzung unseres knappen Bodens und die Zerstörung natürlicher Schönheit des Landes", so 1966 H. Gutersohn als Kommissionspräsident der Eidgenössischen Expertenkommission für Fragen der Landesplanung. Diese Kommission wurde 1963 vom Bundesrat eingesetzt, nachdem die Bodenrechtsabstimmung negativ ausfiel, die Probleme um die Nutzung des Bodens und die geordnete Besiedlung des Landes jedoch blieben. Die Arbeit dieses Gremiums mündete im Verfassungsartikel $22^{\text {quater }}$, der 1969 mit großer Mehrheit von Volk und Ständen angenommen wurde.

\section{Art. $22^{\text {quater }}$ \\ ' Der Bund stellt auf dem Wege der Gesetzgebung Grund- sätze auf für eine durch die Kantone zu schaffende, der zweckmäßigen Nutzung des Bodens und der geordneten Besiedelung des Landes dienende Raumplanung. \\ ${ }^{2}$ Er fördert und koordiniert die Bestrebungen der Kantone und arbeitet mit innen zusammen. \\ ${ }^{3}$ Er berücksichtigt in Erfüllung seiner Aufgaben die Erforder- nisse der Landes-, Regional- und Ortsplanung.}

1943, also lange vor dieser Abstimmung, die 1969 den Weg zu einem Bundesgesetz über die Raumplanung ebnete, wurde in Zürich ein «Institut für Landesplanung» gegründet und dem Geographischen Institut der Eidgenössischen Technischen Hochschule angegliedert. 1961 verselbständigte es sich dann unter dem Namen «Institut für Orts-, Regional- und Landesplanung». Dort begannen um 1967 die - für die zweite Periode richtungsweisenden - Arbeiten an den landesplanerischen Leitbildern.

Die Landesplanung wurde damals, ganz im Sinne der traditionellen Geographie, als etwas Umfassendes, d.h. als zielorientierte Gesamtplanung, verstanden, die sich über das ganze Land erstrecken sollte. Diese Grundvorstellungen fanden im ersten Bundesgesetz

Hans Flückiger, Dr. rer. pol.

Stellvertretender Direktor des Bundesamtes für Raumplanung Bundesamt für Raumplanung, 3003 Bern 
über die Raumplanung ihren Niederschlag. Die in jener Zeit publizierten Landesplanerischen Leitbilder lieferten gewissermaßen das konkrete Anschauungsmaterial für die Auseinandersetzungen um das erste Raumplanungsgesetz. Ebenfalls in dieser zweiten Periode schritten Regierung und Parlament gegen die immer schlimmer werdende Zersiedlung des Landes ein: Mittels Notrechts (Bundesbeschluß über dringliche Maßnahmen auf dem Gebiete der Raumplanung, vom 17. März 1972) mußte landesweit die Landschaft vor einer ungeordneten Überbauung bewahrt werden.

Eine ganze Anzahl von Kantonen betrachtete indessen die Pflicht zur Bezeichnung von Schutzgebieten als obrigkeitliche Maßnahme. Nicht zuletzt unter diesem Eindruck erwuchs dem ersten Bundesgesetz über die Raumplanung ernste Opposition, die am Ende auch erfolgreich war. Diese Opposition rügte den zentralistischen Charakter einer leitbildorientierten Raumplanung. Sie fürchtete eine zu starke Einflußnahme "Berns» in kantonale Hoheit - ein Attribut, das im traditionell föderalistisch ausgerichteten Bundesstaat Schweiz jedes Gesetz zum Scheitern verurteilt. Trotzdem - namentlich in den geschmähten Leitbildberichten wurde viel wertvolle Arbeit geleistet, auch von der Geographie. Dementsprechend groß war dann natürlich die Enttäuschung nach der verlorenen Volksabstimmung - in der Geographie, aber nicht nur dort. Geblieben sind aus jener Zeit reiche Erfahrungen und eine ganze Anzahl Grundlagenberichte, zu wesentlichen Teilen aus der Hand von Geographen, die auch heute ihre Gültigkeit nicht verloren haben: Klimaeignungskarte für die Landwirtschaft, $1: 200000$ (1977), Raumtypisierung nach geographischen Gesichtspunkten als Grundlage der Raumplanung auf höherer Stufe (1974).

Unmittelbar nach der Ablehnung des ersten Raumplanungsgesetzes 1976 begann eine tiefgreifende Neuorientierung in der Raumplanung. Sie führte weg von einer zielorientierten Gesamtplanung Schweiz - hin $\mathrm{zu}$ einem problembezogenen Ansatz mit begleitendem Zielsetzungsproze $\beta$, der durch geeignete Verfahren und Instrumente in den Kantonen sichergestellt werden sollte.

Damit hob die dritte Phase der schweizerischen Raumplanung an. Im nunmehr rechtskräftigen Raumplanungsgesetz sticht die klare Aufgabenteilung zwischen Bund und Kantonen hervor. Der Bund hat sich auf eine Grundsatz-Gesetzgebung zu beschränken, die Planungshoheit liegt ganz eindeutig bei den Kantonen, also der mittleren Ebene in der föderalistischen Hierarchie unseres Landes. Die Hauptaufgabe des Bundes ist somit die Abstimmung bundeseigener raumwirksamer Tätigkeiten sowie die Koordination der kantonalen Richtplanungen. Die Nutzungsplanung ist Sache der Kantone. Materiell zusammengehalten wird dieses Planungssystem durch ein konkre- tes Wertgefüge, das in den Ziel- und Grundsatzartikeln des Raumplanungsgesetzes verankert ist.

Dieses neue Planungsverständnis verlangt ein Umdenken, insbesondere von den Pionieren der Raumplanung in der Schweiz - also auch von den Geographen. Studien und Untersuchungen mit gesamtheitlichem Ansatz werden zwar noch als Planungsgrundlagen gebraucht, doch das Hauptgewicht planerischer Arbeit liegt auf der Lösung von Konflikten, die durch konkurrierende Nutzungsansprüche an den Boden entstehen. Damit ist die Geographie aufgerufen, ihre Aktivitäten vermehrt auf aktuelle Probleme auszurichten. Im Zusammenhang mit der Anwendung der Planungsgrundsätze bei Richt- und Nutzungsplanungen bestehen beispielsweise große Wissenslücken. Hier tut sich der Geographie - aber nicht nur ihr - ein neues, breites Feld praxisorientierter wissenschaftlicher Tätigkeit auf. Es liegt nun an ihr, diese Herausforderung anzunehmen.

\section{Was erwartet die Raumplanung heute von der Geographie?}

Aus dem Vorangegangenen geht eines hervor: Die Raumplanung hat sich heute als eigenständige Staatsaufgabe etabliert. Sie hat ein Selbstverständnis entwikkelt und ist - im großen ganzen - in der Lage, ihre Bedürfnisse an Wissenschaft und Forschung zu formulieren. Dabei spricht sie verschiedene Hochschuldisziplinen an, nämlich all jene, die sich in irgendeiner Weise mit Elementen und Vorgängen im Raum befassen, darunter natürlich auch die Geographie. Doch es läßt sich nicht verkennen, daß die «grosse Zeit» der Geographie in der Raumplanung in der ersten Phase stattfand. In der zweiten Periode ging es dann gewissermaßen um das «Erbe» der Geographie, um den Anspruch auf ganzheitliche Betrachtung und Planung des Raumes. In der heutigen dritten Phase, so scheint es, hat die Geographie kaum mehr entscheidende Akzente in der Raumplanung gesetzt. Wohl erregen hie und da, zumindest in Fachkreisen, geographische Studien ein gewisses Aufsehen. Von einer Leaderstellung der Geographie in der Raumplanung kann allerdings nicht mehr gesprochen werden.

Die Geographie hat sich heute, so erscheint es einem Außenstehenden, stark auf eng umgrenzte Teilbereiche spezialisiert. Dabei gerät ausgerechnet jene, als spezifisch geographisch bezeichnete Denkweise in räumlichen Dimensionen ins Hintertreffen.

Die Schweizer Raumplanung hat bis heute zahlreiche im besonderen Interessenbereich der Geographie liegende Fragen aufgeworfen oder mitformuliert und Studien zu deren Beantwortung unterstützt. Im Vordergrund stehen dabei:

- Abklärungen über die Bedeutung der natürlichen Lebensgrundlagen (Art. 1 Abs. 2 RPG) für die 
Planung und Abstimmung raumwirksamer Tätigkeiten auf den verschiedenen Ebenen unseres Staatswesens;

- Erfassung und Charakterisierung von Landschaften mit Streusiedlungen und Analyse ihrer Veränderungstendenzen;

- Erfassung und Analyse der bevölkerungs- und nutzungsmäßigen Entwicklung der Schweiz («Raumbeobachtung Schweiz»);

- Die drei Nationalen Forschungsprogramme

- "Man and Biosphere» (MAB);

- "Regionalprobleme» und neu

- «Nutzung des Bodens».

In allen Bereichen arbeiten Geographen aktiv mit oder sind zur Mitarbeit aufgerufen. Trotzdem erscheint dem Außenstehenden mehr Forschungsengagement im Bereiche der Raumplanung durchaus möglich - und erwünscht. Zu oft, so der Eindruck, wendet die Geographie ihren forschenden Blick zurück in die Vergangenheit, anstatt ihn auf aktuelle raumplanerische Probleme zu lenken.

- Dabei sind, so scheint mir, zahlreiche Problembereiche für eine geographische Erforschung geradezu prädestiniert, vorausgesetzt, diese Fragen werden auch von der Geographie als solcher gesehen und dementsprechend als Anliegen anerkannt - ohne direkte Aufträge oder Anstöße von außen. Analysieren wir doch einmal das Raumplanungsgesetz vom 22.Juni 1979 mit «forschungshungrigen» Augen:

Die Planungsgrundsätze (Art. 3 RPG) sind zwar deutlich formuliert, aber nicht ohne weiteres und direkt in die Praxis umzusetzen. Sie müssen im Einzelfall bewertet, abgewogen und umgesetzt werden können. Die planenden Instanzen in Bund, Kantonen und Gemeinden wären bei ihren raumwirksamen Tätigkeiten der Geographie um klärende Kriterien sicher dankbar, etwa in folgenden Fällen:

- «Die Landschaft ist zu schonen. Insbesondere sollen...

Siedlungen, Bauten und Anlagen sich in die Landschaft einordnen" (Art. 3 Abs. 2 Bst. b RPG).

Nach welchen Kriterien soll zum Beispiel die Einordnung baulicher Anlagen in eine Landschaft bewertet werden?

- "Die Landschaft ist zu schonen. Insbesondere sollen...

Naturnahe Landschaften und Erholungsräume erhalten bleiben" (Art. 4 Abs. 2 Bst. d RPG).

Welche Kriterien sind zum Beispiel maßgebend, um Einwirkungen auf Landschaften und Erholungsräume zu bewerten? Wie bemißt sich der Wert naturnaher Landschaften und Erholungsräume? Wie ist er der Bevölkerung besser verständlich zu machen?

- «Für die öffentlichen oder im öffentlichen Interesse liegenden Bauten und Anlagen sind sachgerechte Standorte zu bestimmen" (Art. 3 Abs. 4 PRG).
Welche Entscheidungsgrundlagen könnte die Geographie für die Bestimmung sachgerechter Standorte beibringen, wenn gleichzeitig regionale Bedürfnisse berücksichtigt und störende Ungleichheiten abgebaut werden sollen?

Der Richtplan nach Raumplanungsgesetz verlangt nicht nur neue Überlegungen zur Erfassung des Planungs- und Abstimmungsstandes, er verlangt mindestens teilweise - auch nach neuen kartographischen Darstellungstechniken. Offen sind zum Beispiel die Fragen der Darstellung von Konflikten oder von gleichen Elementen mit unterschiedlichem Planungsstand im Hinblick auf die angestrebte Entwicklung.

Nach Artikel 2 der Verordnung über die Raumplanung (RPV) stellen die Behörden fest, «wie sich die raumwirksamen Tätigkeiten auswirken". Für die Erfüllung dieses Auftrages fehlen den Behörden aller Stufen noch wichtige Grundlagen. Wenn etwa der Bund seine Konzepte und Sachpläne (Art. 13 RPG) erstellt, muß er mehr über die Auswirkungen seiner Vorhaben wissen, als dies heute der Fall ist. Was sagen zum Beispiel Geographen zur neuen Haupttransversalen (NHT) der Schweizerischen Bundesbahnen? Wie sind aus ihrer Sicht die verschiedenen Varianten zu bewerten? Wie sieht das Vorhaben im Zusammenhang mit dem übrigen öffentlichen Verkehr bzw. dem Privatverkehr aus?

Auf der Stufe der Nutzungsplanung schließlich geht es heute nicht mehr ausschließlich um eine parzellengenaue Baugebietsfestlegung, bisher häufig eine Domäne von Architekten und Bauingenieuren. Das Raumplanungsgesetz verlangt eine Nutzungsplanung des ganzen Gemeindegebietes und weist Landwirtschafts- und Schutzzonen grundsätzlich gleiches Gewicht zu wie den Bauzonen. Eine Nutzungsplanung nach RPG setzt also ein ganzheitliches Raumverständnis voraus und spricht damit natürlich Geographen besonders an. An ihnen ist es, die spezifischen Vorteile dieser Betrachtungsweise sichtbar zu machen. Die Raumplanung wäre für entsprechende Arbeiten und Hinweise sicher sehr dankbar.

Die Geographie hat in der Pionierphase der Schweizer Raumplanung an vorderster Front gestritten und Bedeutendes geleistet. Seither verdanken wir ihr immer wieder wichtige Beiträge. Allerdings, an der Weiterentwicklung der Raumplanung in der Schweiz war die Geographie recht zurückhaltend beteiligt, so jedenfalls der Eindruck eines Außenstehenden. Inwieweit diese Beurteilung zutrifft und wo allenfalls die Ursachen liegen, das sind Fragen, die sich an die "angewandten Geographen" richten. An ihnen ist es, dieser Auffassung zu widersprechen - oder dann für ihr Fach die notwendigen Schlußfolgerungen $\mathrm{zu}$ ziehen. In diesem Sinne verstehe ich die Schilderung der Geschichte der Raumplanung sowie der Rolle, welche die Geographie in ihr spielt, als Anstoß zu einer fruchtbaren Diskussion. 